\title{
EINIGE BEMERKUNGEN ZU DEN LIEFERGEBIETEN UND SEDIMENTATIONSRAUMEN DER LOSSE IM RAUM MARBURG/LAHN AUF GRUND TONMINERALOGISCHER UNTERSUCHUNGEN *
}

\author{
Mit 5 Abbildungen, 2 Bildern und 2 Tabellen
}

\section{Klaus Heine}

Summary: Some observations on the source districts and sedimentation areas of the loesses in the Marburg/Lahn area on the basis of clay mineral investigations

$\mathrm{X}$-ray diagrams and electron microscope prints of the clay fractions of various Würm loesses in the Marburg area show surprising differences with regard to the set of clay minerals which could not be solely caused by pedochemical processes after loess accumulation, but must be attributed to processes operating in different source areas. An important part of the loess material was blown out in close proximity to the region of deposition. Qualitative differences in clay mineral linkages indicate the following main source areas: the loesses on the eastern edge of the Rhine Massif come from the slopes of the massif and the gravel floors of the streams debouching from the mountains; the loesses of the middle Lahn valley were blown out of the Lahn gravels; the loess material of the northern Amöneburg Basin comes largely from the gravels of the Ohmtal Depression, and the loesses of the southern Amöneburg Basin were blown from the lower Vogelsberg and from the valleys of nearby rivers and streams. A comparison of the results of the loess investigations with those of various flood plain silts confirms the view that the blowing out and accumulation of the loesses must have taken place within a very small area.

In einem auffälligen Zusammenhang mit der Topographie steht die Verbreitung des Lösses in der Marburger Landschaft. Der Löß findet sich vorwiegend an den Hängen der kleineren Täler des östlichen Rheinischen Schiefergebirges und des Marburger Buntsandsteingebietes, und zwar auf den westlichen, nach Osten orientierten Seiten. Im Amöneburger Becken werden weite Teile von of $t$ recht mächtigen Lössen bedeckt; doch auch hier kommen Lösse hauptsächlich westlich der Ohm vor, während sie an den sanft nach Osten ansteigenden Hängen fast ganz fehlen. Bemer- kenswert ist auch das Fehlen größerer Lößablagerungen an den Hängen des Lahntales im Buntsandsteingebiet.

Auf Grund der Lößbedeckung der nach Osten orientierten Hänge schließt BlumE $(1949,66)$ einen Transport des Materials aus Westen, und zwar vor allem aus den Flußschottern des Rheins, nicht aus, doch er stellt gleichzeitig die Frage, wieso bei westlichen Winden die Täler des Schiefergebirges frei von Löß blieben, die Lößbedeckung erst hart westlich Marburgs einsetzt und im Osten des Marburger Buntsandsteingebietes im Amöneburger Becken eine größere Mächtigkeit erlangt. Die Verteilung des Lösses in dem hier betrachteten Raum läßt sich mit einem Ferntransport des äolischen Materials aus Westen (vgl. Woldstedt 1961, 185 f.) nur teilweise in Einklang bringen. Ein Ferntransport aus einem anderen Gebiet, wie z. B. aus dem der norddeutschen Moränen und Sander, erscheint noch weniger den Verhältnissen zu entsprechen.

Tonmineralogische Untersuchungen an verschiedenen Lössen hinsichtlich der Tonmineralgarnitur zeigen jedoch auffällige Unterschiede, die nicht allein durch pedochemische Prozesse nach der Lößakkumulation verursacht werden können, sondern aus denen auf verschiedene Liefergebie te geschlossen werden darf. Bei einem Vergleich der Untersuchungsergebnisse der Lösse mit denen verschiedener Auelehme

* Herrn Professor Dr. Dr. E. Mückenhausen vom Bodenkundlichen Institut der Universität Bonn möchte ich auch an dieser Stelle für sein großes Entgegenkommen danken, daß er mir die Geräte seines Institutes zur Verfügung stellte und veranlaßte, daß Frl. Bödewadt die elektronenmikroskopischen Aufnahmen für mich ausführte. 


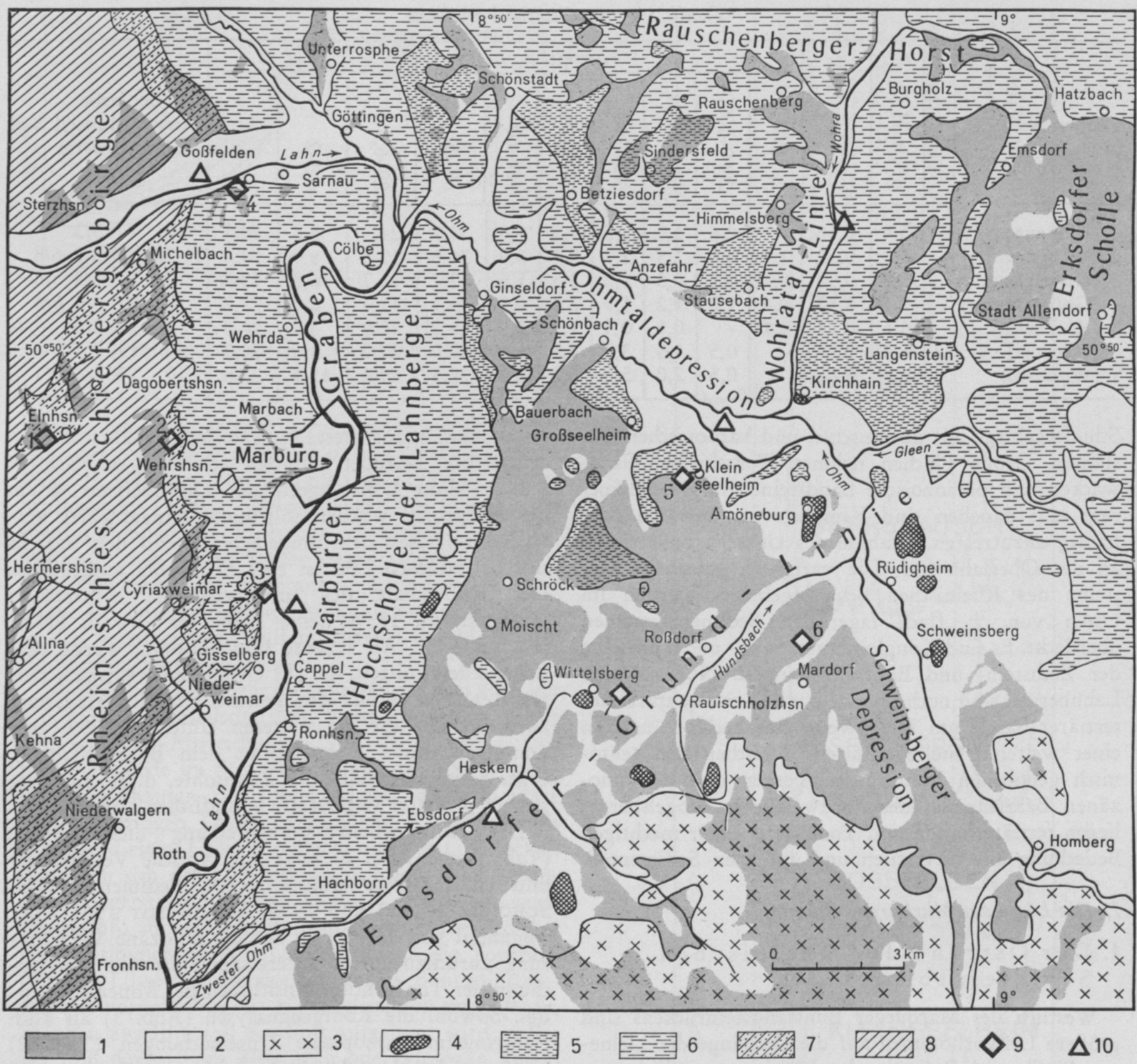

Abb. 1: Geologische Übersichtskarte des Amöneburger Beckens und des Lahntales bei Marburg (z. T. nach HöLtiNG und STENGEL-RUTKOWSKI 1964)

1 Löß; 2 Quartär u. sedimentäres Tertiär; 3 Decken; 4 Durchbrüche (3 u. 4 basaltisches Tertiär); 5 Oberer Buntsandstein (Röt); 6 Mittlĕrerỉ Buntsandstein; 7 Perm; 8 Karbon u. ältere Formationen; 9 Lößproben; 10 Auelehmproben

wird deutlich, daß das Auftreten ganz bestimmter Tonmineralvergesellschaftungen für kleinräumige Landschaftselemente typisch zu sein scheint.

Bei einer Abgrenzung verschiedener Lößprovinzen auf Grund ihrer mineralogischen Eigenschaften bietet die Bestimmung der Tonminerale große Vorteile gegenüber anderen Verfahren (vgl. KallenBaCH 1966). Für Schwermineralanalysen beispielsweise ist ein Korndurchmesser von $0,06 \mathrm{~mm}$ bereits die untere Grenze. Erst von dieser Grenze an sind mit Hilfe der allgemein angewandten Methoden die einzelnen Minerale sicher zu identifizieren (vgl. GuentHer 1961, 12). Gerade die gröberen Bestandteile der Lösse
$(>0,06 \mathrm{~mm})$ aber stammen oft aus unmittelbarer Nähe des Ablagerungsgebietes; daher und auf Grund ihres höheren spezifischen Gewichtes und des ihnen eigenen Verhaltens bei der Ausblasung, dem Transport und der Anwehung erlauben die Ergebnisse einer Schwermineralanalyse keine allgemeingültigen Schlüsse im Zusammenhang mit dem oben angeschnittenen Problemkreis.

Da sowohl die Lösse wie auch die Auelehme eine enge Abhängigkeit von dem petrographischen Aufbau der Marburger Landschaft zeigen, soll dieser kurz skizziert werden (Abb. 1). Im Westen erstreckt sich das in viele einzelne Kuppen aufgelöste Rheinische 
Tabelle 1: Analysendaten der Lösse

\begin{tabular}{|c|c|c|c|c|c|c|c|c|c|c|c|c|c|}
\hline \multirow[b]{2}{*}{ Proben-Nr. } & \multirow[b]{2}{*}{ 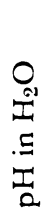 } & \multirow[b]{2}{*}{ 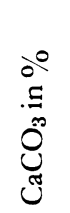 } & \multicolumn{10}{|c|}{ Korngröße in $\mu$ in Gew.-\% } & \multirow[b]{2}{*}{$\begin{array}{l}\text { Bodenfarbe } \\
\text { (nach Munsell Soil } \\
\text { Color Charts) }\end{array}$} \\
\hline & & & §̊ి & 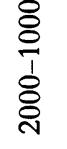 & $\begin{array}{l}8 \\
0 \\
0 \\
0 \\
0\end{array}$ & $\begin{array}{l}\stackrel{0}{\pi} \\
\text { î. } \\
\text { o } \\
\text { n }\end{array}$ & 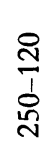 & 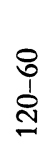 & $\begin{array}{l}\text { ते } \\
\text { రి }\end{array}$ & $\begin{array}{l}\text { no } \\
\text { î } \\
\text { లి }\end{array}$ & $\begin{array}{l}\hat{N} \\
n \\
0 \\
0\end{array}$ & $\stackrel{v}{V}$ & \\
\hline 1 (Elnhausen) & 5,4 & 0,16 & - & 0,6 & 1,1 & 1,5 & 1,3 & 1,8 & 39,7 & 24,3 & 5,6 & 24,1 & $10 Y R 5 / 3$ \\
\hline 2 (Wehrshsn.) & 6,0 & 0,52 & 1,5 & 2,0 & 1,5 & 2,7 & 2,4 & 3,9 & 34,5 & 19,5 & 8,0 & 24,0 & 7,5 YR $6 / 6-6 / 8$ \\
\hline 3 (Gisselberg) & 7,8 & 0,94 & 0,1 & 0,2 & 0,5 & 1,2 & 1,5 & 5,6 & 52,7 & 14,7 & 6,2 & 17,3 & 7,5 YR $5 / 4-5 / 6$ \\
\hline 4 (Goßfelden) & 7,4 & 1,53 & 0,1 & 1,0 & 1,2 & 1,9 & 2,7 & 6,7 & 45,1 & 11,4 & 6,9 & 23,0 & 7,5 YR $6 / 4-6 / 6$ \\
\hline 5 (Kl. Seelh.) & 6,1 & 0,71 & 0,1 & - & 0,1 & 0,4 & 1,1 & 2,7 & $55,2 !$ & 12,9 & 3,2 & 24,3 & 7,5 YR $6 / 6$ \\
\hline 6 (Mardorf) & 5,3 & 0,27 & - & 0,5 & 1,5 & 3,0 & 2,5 & 6,0 & 29,0 & 20,2 & $8,6 i$ & 28,7 & $10 \quad$ YR $7 / 4$ \\
\hline 7 (Wittelsb.) & 7,9 & 0,85 & - & 0,5 & 2,0 & 5,5 & 5,0 & 4,0 & $25,5^{\top}$ & 19,4 & 11,1 & 27,0 & YR $6 / 4-7 / 4$ \\
\hline
\end{tabular}

Schiefergebirge mit devonischen und karbonischen Gesteinen. Große Flächen nehmen Tonschiefer, Grauwacken und paläozoische Sandsteine und Konglomerate ein; daneben sind Kieselschiefer und Quarzite häufig anzutreffen. Diabase des Unterkarbons treten an der Oberlahn auf. Die variszisch gefalteten Gesteine des Rheinischen Schiefergebirges werden im Osten von \pm flach lagernden Sedimentgesteinen überdeckt. Es sind Konglomerate, Sand- und Tonsteine der Zechstein- und Buntsandsteinzeit. Nur auf den Lahnbergen sind noch unbedeutende Reste quarzreicher tertiärer Sande am Fuß zweier Basaltschlote und an einer weiteren Stelle erhalten. Zwischen den tektonisch gehobenen Buntsandsteinbergen und den miozänen Basaltdecken des nordwestlichen Vogelsberges liegen tertiäre Sande und Tone unter einer mächtigen Bedeckung aus fluviatilen und äolischen Sedimenten.

\section{Die Lösse des Marburger Raumes}

1. Der Ostrand des Rheinischen $\mathrm{Sch}$ iefergebirges

Westlich des Marburger Buntsandsteinrückens sind größere Lößvorkommen auf die Osthänge der kleineren Fluß- und Bachtäler beschränkt, so südlich von Michelbach, westlich von Dagobertshausen, Elnhausen und Wehrshausen, nördlich von Cyriaxweimar und in den Talzügen $z$ wischen Hermershausen, Allna und Kehna. In zwei Aufschlüssen wurden Lößproben entnommen.

Die Probe 1 entstammt einem Lößprofil oberhalb des Grauwackensteinbruchs an der Straße von Elnhausen nach Dilschhausen etwa $1 \mathrm{~km}$ westlich Elnhausen. Im Zusammenhang mit den Steinbrucharbeiten war im Herbst 1968 ein Lößprofil von ca. 5 m Mächtigkeit aufgeschlossen. Im Liegenden befand sich ein mit kleinen Gesteinsbruchstücken durchsetzter, stark verwitterter (25,9\% Tongehalt), fast völlig entkalkter Löß von hell gelblich-brauner Farbe (10 YR 5/4 bis 6/4), den ein schmales Solifluktionsband aus groben Steinen $(\phi$ bis $14 \mathrm{~cm})$ der oberhalb am Hang anstehenden Grauwacken und Kieselschiefer durchzieht.
Die Bodenbildung dieses Horizontes ist wahrscheinlich eemzeitlich. Darüber befinden sich ca. $4 \mathrm{~m}$ mächtige würmzeitliche Lößablagerungen. Die obersten $40 \mathrm{~cm}$ des Lößprofils wurden solifluidal hangabwärts verlagert; zwischen dem umgelagerten und dem sich in situ befindlichen Löß ist eine Steinsohle ausgebildet. Der Kalkgehalt des unverwitterten Würmlösses schwankt um $15 \%$, seine Farbe ist schwach braun (10 YR 6/3). Als rezente Bodenbildung wurde eine Braunerde ermittelt, die auf Grund einer schwach sauren Reaktion bereits eine gewisse Kolloidverlagerung aufweist. Die für die weiteren Untersuchungen entnommene Probe 1 entstammt dem Bv-Horizont ${ }^{1}$ ), etwa $5 \mathrm{~cm}$ unterhalb der Steinsohle, d. h. aus dem sich an primärer Lagerstätte befindlichen Löß.

Die Korngrößenzusammensetzung dieser Probe (Tab. 1 und Abb. 2) zeigt das typische Verteilungsbild eines äolisch transportierten Sedimentes (vgl. Schönhals 1953 und 1955; WoldSTEDT 1961); der Tonanteil von $24,1 \%$ ist auf die holozäne Verwitterung zurückzuführen. Interessanter ist die Untersuchung der Tonfraktion hinsichtlich des Mineralbestandes. Sowohl die Röntgenanalysen (Abb. 3) als auch elektronenmikroskopische Untersuchungen (Tab. 2) ergaben als Hauptbestandteile Hydroglimmer und daneben Quarz; als Nebenbestandteile treten Illit und Kaolinit auf; Montmorillonit befindet sich in dieser Probe lediglich in Spuren. Daß die Tonmineralgarnitur von Probe 1 nicht allein das Produkt der nacheiszeitlichen Verwitterung ist, $d$. h. von dem

$\left.{ }^{1}\right)$ Die Proben wurden jeweils den Bv-Horizonten von Würmlössen entnommen, da hier infolge der Verwitterung der ursprüngliche Mineralbestand der Lößfraktion 60-20 $\mu$ zu einem gewissen Teil in Tonminerale umgebildet wurde. Eine Probenahme aus den C-Horizonten empfiehlt sich nicht, da in ihnen eine postsedimentäre Tonmineralneubildung kaum zu erwarten ist. Die Tonminerale der C-Horizonte sind größtenteils präsedimentärer Entstehung; sie sind daher nicht unbedingt charakteristisch für den Mineralbestand der eigentlichen Lößfraktion 60-20 $\mu$ und damit auch nicht immer repräsentativ für den mengenmäßig größten Anteil des Lößmaterials. 


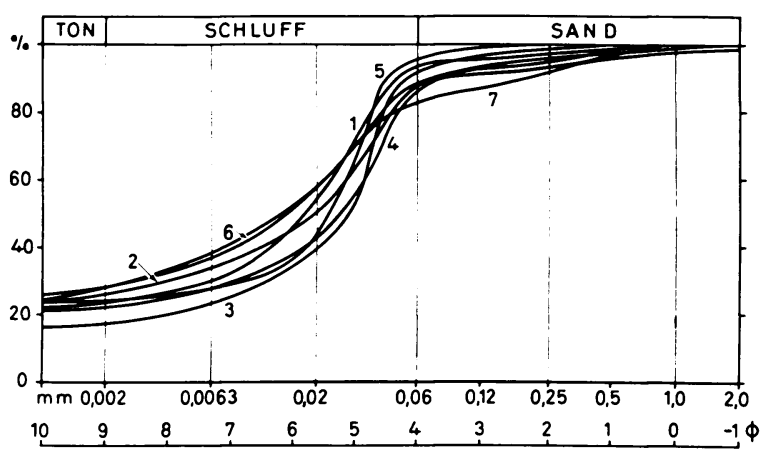

$A b b$. 2: Kornsummenkurven der Lösse (Bv-Horizonte)

Relief, dem Klima, dem Grundwasser, der Vegetation, der menschlichen Arbeit oder anderen Faktoren abhängt, zeigt ein Vergleich mit den anderen hier beschriebenen Proben verschiedener Würmlösse, die ebenfalls einem Bv-Horizont entnommen wurden. Vielmehr muß - wie weiter unten aufzuzeigen ist eine Anwehung aus bestimmten Liefergebieten und daher ein unterschiedlicher Mineralbestand der Lösse in den verschiedenen Teillandschaften des Marburger Raumes angenommen werden.

Hinsichtlich der Korngrößenverteilung wie auch der Tonmineralvergesellschaftung weist die Probe 2 nur unbedeutende Abweichungen von der Probe 1 auf. Probe 2 wurde im Straßeneinschnitt ca. $400 \mathrm{~m}$ westlich Wehrshausen dem Bv-Horizont eines mehrere Meter mächtigen Lösses entnommen. Allerdings läßt sich bei diesem Lößvorkommen nicht mit Sicherheit sagen, ob sich der Löß hier an primärer oder sekundärer Lagerstätte befindet. Handelt es sich um einen Primärlöß, so veranschaulichen die mit der Probe 1 übereinstimmenden Analysendaten, daß die Tonfraktion der Lösse westlich des Marburger Buntsandsteingebietes vornehmlich aus Hydroglimmern und Quarz, daneben aus Illit und Kaolinit aufgebaut wird; sollte der Löß dagegen im Pleistozän oder Holozän umgelagert worden sein, so zeigen die Ergebnisse darüber hinaus, daß eine nach der Sedimentation erfolgte Verlagerung keinen großen Einfluß auf die Zusammensetzung der Tonminerale haben kann.

\section{Das Mittellahntal}

Größere Lößakkumulationen fehlen im Tal der Mittellahn im Bereich des Buntsandsteins. Nur auf den weniger steil geböschten Flächen pleistozäner Flußterrassen werden das anstehende Gestein und fluviatile Lahnschotter mitunter von Solifluktionsdecken aus Buntsandsteinmaterial und Löß bedeckt. Lediglich nördlich Gisselberg, zwischen Ockershausen und Marburg und südlich des weißen Steins bei Wehrda sind an dem westlichen Lahntalrand Würmlösse erhalten. An der Ockershäuser Allee in Marburg fand E. JacobshageN (1933) im Löß eine reiche

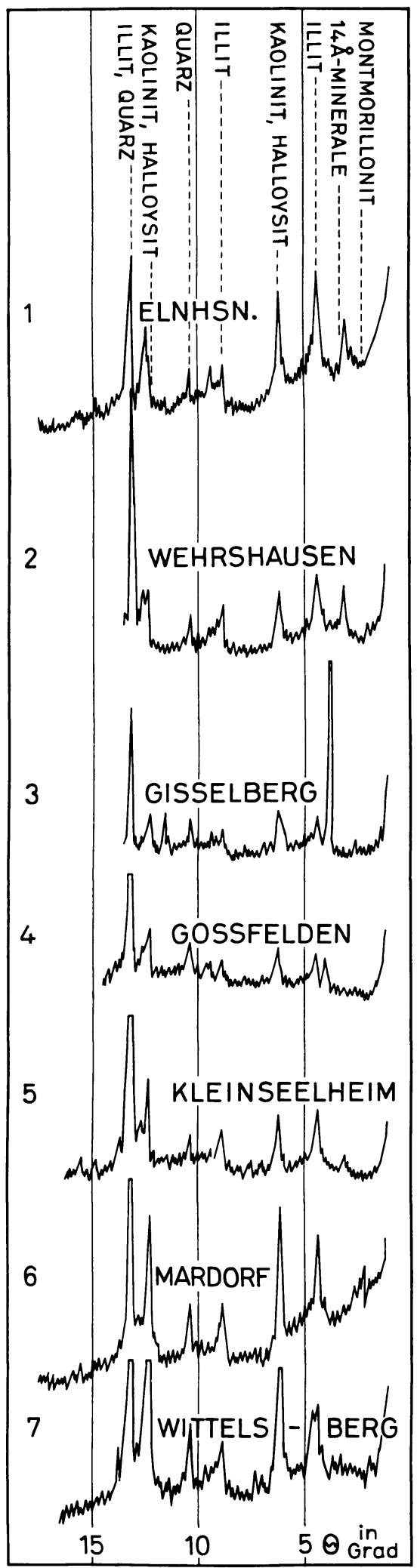

Abb. 3: Röntgendiagramme der Lösse (Bv-Horizonte) 
Tabelle 2: Ergebnisse der elektronenmikroskopischen Untersuchungen

\begin{tabular}{|c|c|c|c|c|}
\hline Proben-Nr. & Hauptbestandteile & Nebenbestandteile & Spuren & Untersuchungs- $\mathrm{Nr}$. \\
\hline 1 (Elnhausen) & $\begin{array}{l}\text { Hydroglimmer } \\
\text { Quarz }\end{array}$ & $\begin{array}{l}\text { Illit } \\
\text { Kaolinit }\end{array}$ & Montmorillonit & $172 / 68$ \\
\hline 2 (Wehrshausen) & $\begin{array}{l}\text { Hydroglimmer } \\
\text { Quarz }\end{array}$ & $\begin{array}{l}\text { Illit } \\
\text { Kaolinit }\end{array}$ & Montmorillonit & $169 / 68$ \\
\hline 3 (Gisselberg) & $\begin{array}{l}\text { Illit } \\
\text { Hydroglimmer }\end{array}$ & $\begin{array}{l}\text { Kaolinit } \\
\text { Quarz }\end{array}$ & $\begin{array}{l}\text { Montmorillonit } \\
\text { Sarospatakit } \\
\text { Sepiolith } \\
\text { Amesit }\end{array}$ & $174 / 68$ \\
\hline 4 (Goßfelden) & $\begin{array}{l}\text { Hydroglimmer } \\
\text { Quarz }\end{array}$ & $\begin{array}{l}\text { Illit } \\
\text { Kaolinit }\end{array}$ & Montmorillonit & $168 / 68$ \\
\hline 5 (Kl. Seelheim) & $\begin{array}{l}\text { Illit } \\
\text { Hydroglimmer }\end{array}$ & $\begin{array}{l}\text { Montmorillonit } \\
\text { Kaolinit } \\
\text { Quarz }\end{array}$ & $\begin{array}{l}\text { Halloysit } \\
\text { Amesit } \\
\text { Eisenminerale }\end{array}$ & $173 / 68$ \\
\hline 6 (Mardorf) & $\begin{array}{l}\text { Illit } \\
\text { Hydroglimmer } \\
\text { Kaolinit }\end{array}$ & $\begin{array}{l}\text { Montmorillonit } \\
\text { Quarz }\end{array}$ & $\begin{array}{l}\text { Halloysit } \\
\text { Eisenminerale } \\
\text { Amesit }\end{array}$ & $170 / 68$ \\
\hline 7 (Wittelsberg) & $\begin{array}{l}\text { Illit } \\
\text { Kaolinit } \\
\text { Hydroglimmer }\end{array}$ & $\begin{array}{l}\text { Montmorillonit } \\
\text { Quarz }\end{array}$ & $\begin{array}{l}\text { Halloysit } \\
\text { Eisenminerale } \\
\text { Amesit }\end{array}$ & $171 / 68$ \\
\hline
\end{tabular}

Wirbeltierfauna mit Rhinoceros lenensis (Wollhaarnashorn), Megaceros giganteus (Riesenhirsch), Bison priscus, Mammonteus primigenius und Rangifer. Dieser Aufschluß ist seit Jahrzehnten verbaut.

Etwa $1 \mathrm{~km}$ nördlich Gisselberg bedeckt ein rund $4 \mathrm{~m}$ mächtiges würmzeitliches Lößprofil die rißzeitlichen Lahnterrassenschotter. Im liegenden Teil des Profils zeigen sandige Schlieren an, daß zu Beginn der Lößakkumulation fließerdeartige Bewegungen stattgefunden haben müssen. Der unverwitterte Löß hat eine hellbraune Farbe (10 YR 6/3) und einen Kalkgehalt von $13-16 \%$; darin fand Huckriede (frdl.mdl. Mitt. von Herrn Prof. Dr. R. Huckriede, Geol. Inst. Marburg) verschiedene Nacktschnecken neben Pupilla muscorum und Succinea oblonga, was auf ein relativ feuchtes Klima während der Lößsedimentation hinweist. Probe 3 enthält Material des Bv-Horizontes einer Braunerde, die sich auf diesem Löß im Holozän bildete.

Die Korngrößenverteilung der Probe 3 zeigt keine wesentlichen Unterschiede zu den Analysendaten bzw. den Summenkurven der Proben 1 und 2 aus dem Gebiet westlich des Marburger Rückens. Doch die röntgenographischen und elektronenmikroskopischen (Bild 1) Untersuchungen der Tonfraktion von Probe 3 lassen erkennen, daß hier Illite (neben den Hydroglimmern) als Hauptbestandteile und nicht mehr als Nebenbestandteile auftreten. Auch ist der Quarzanteil wesentlich geringer als in den erwähnten Proben aus dem Randbereich des paläozoischen Rheinischen Schie- fergebirges. Neben Quarz enthält Probe 3 Kaolinit als Nebenbestandteile, dazu Montmorillonit, Sarospatakit, Sepiolith und Amesit in Spuren.

Bei Goßfelden verläßt die Lahn das Rheinische Schiefergebirge. Aus einem Lößprofil von $4 \mathrm{~m}$ Mächtigkeit auf einer pleistozänen Felsterrasse unmittelbar oberhalb der Talaue südwestlich von Goßfelden stammt die Probe 4. Es handelt sich um Material des nur schwach verwitterten Bv-Horizontes einer rezenten Braunerde. Obgleich dieses Lößvorkommen nicht mehr dem Mittellahntal i. e. S. zuzurechnen ist, so fügen sich doch die Laborbefunde in das Gesamtbild recht gut ein, in der Weise nämlich, daß sie eine Zwischenstellung hinsichtlich der tonmineralogischen Eigenschaften $z$ wischen den Lößproben 1 und 2 des paläozoischen Gesteinsbereichs und der Probe 3 aus dem Mittellahntal einnehmen. Das Röntgendiagramm der Probe 4 ist durch die Reflexe zwischen 10 und $14 \AA$ geprägt und ähnelt damit sehr dem der Probe 3. Die Reflexe zwischen 10 und $14 \AA$ treten doppelt auf; sie deuten auf einen hohen Gehalt an Hydroglimmern, die hier bereits durch die Verwitterungseinflüsse weiter abgebaut sind als in den Proben 1 und 2. Auf die Ursachen für einen verstärkten Glimmerabbau in den Lössen an den Hängen des Mittellahntales werde ich weiter unten eingehen. Das Röntgendiagramm zeigt eine auffällige Ähnlichkeit mit der Probe 3, die elektronenmikroskopischen Untersuchungen dagegen ergaben für die Probe 4 die gleichen Ergebnisse wie für die Proben 1 und 2 (Tab. 2). 


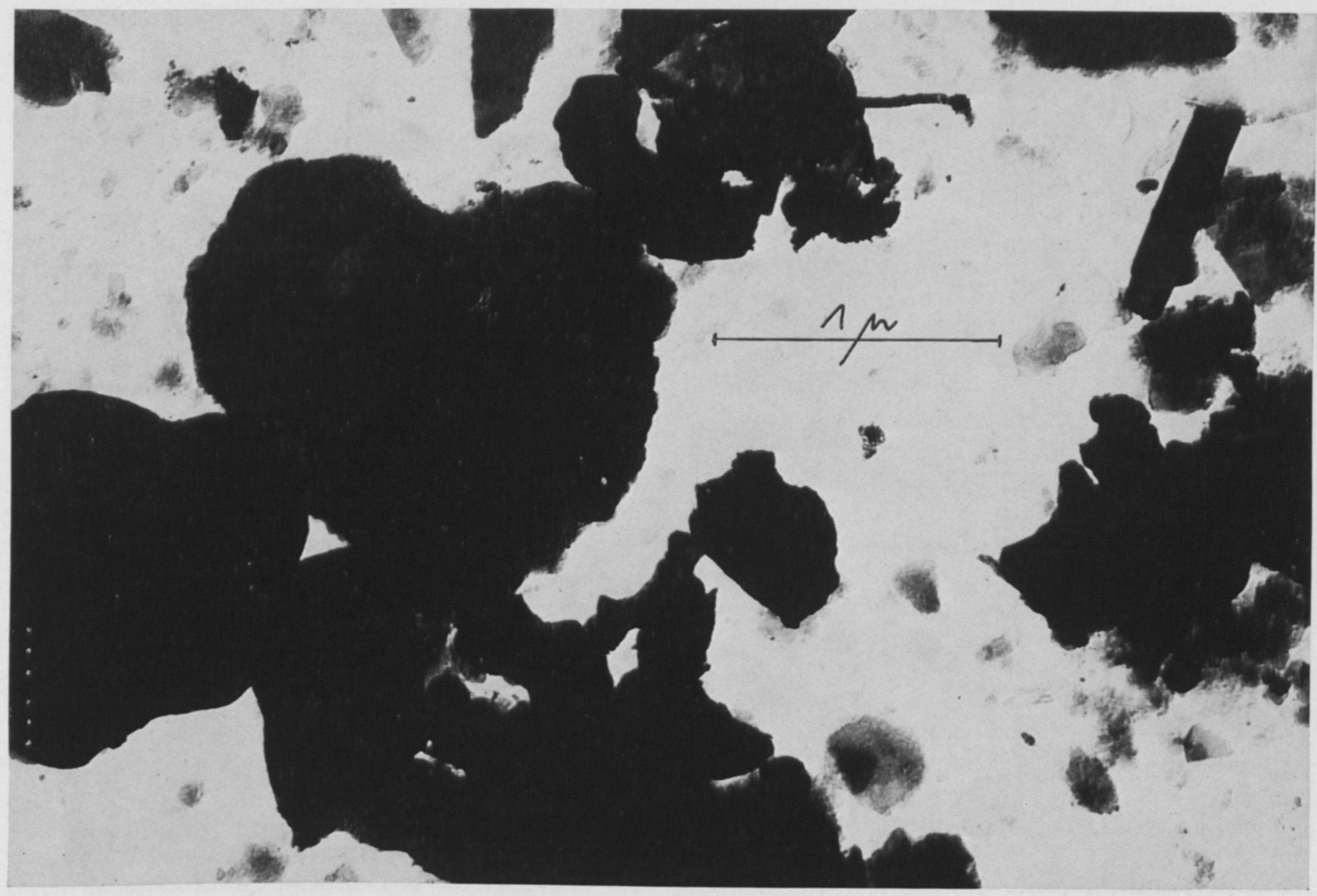

Bild 1: Elektronenmikroskopische Aufnahme der Tonfraktion $(<1 \mu)$ der Probe 3 (Gisselberg). Als Bestandteile sind zu erkennen: Vorwiegend Illit und Hydroglimmer, daneben Kaolinit, Quarz, in Spuren Montmorillonit, Sarospatakit, Sepiolith und Amesit.

3. Das nördliche

A möneburger B ecken

Löß und geschiebefreie Lehme sind im Bereich des Amöneburger Beckens weit verbreitet. Die östlichen Abhänge der Lahnberge sind an ihrem Fuß mit einer oft recht mächtigen Lößdecke überzogen; bei Schröck wurden über $15 \mathrm{~m}$ dicke Lößablagerungen erbohrt (Hölting und Stengel-Rutkowski 1964, 24). Es wurde bereits erwähnt, daß die sanft ansteigenden Hänge östlich des Ohmflusses fast völlig lößfrei sind; aber auch der Anstieg von der Ohmtaldepression zu den Buntsandsteinhöhen im Norden trägt keine nennenswerte Lößbedeckung. Stratigraphische Untersuchungen an den Lössen des Amöneburger Beckens stehen noch aus; nur vereinzelt findet man in der Literatur Hinweise auf einen Wechsel von Sedimentations- und Bodenbildungsphasen in der näheren und ferneren Umgebung Marburgs (vgl. u. a. WEYL 1967, 54 u. 82 ff.; Kockel 1958, 162; BLANCKENHORN 1930, 61).

Stellvertretend für die Lösse aus dem nördlichen Amöneburger Becken soll die Probe 5, die dem BvHorizont eines Würmlösses der Ziegeleigrube südwestlich Kleinseelheim entstammt, betrachtet werden.
Das Bild der Korngrößenverteilung hebt sich nicht von dem der bisher besprochenen Proben ab. Allerdings ist der Anteil der Fraktion 60-20 $\mu$ mit 55,2 \% in Probe 5 größer als in allen anderen. Die Zusammensetzung der Tonminerale zeigt als Hauptbestandteile Illit und Hydroglimmer; sie ähnelt damit auf den ersten Blick der Probe aus dem Lahntal bei Gisselberg, doch bereits das häufigere Auftreten von Montmorillonit neben Kaolinit und Quarz - hier als Nebenbestandteile - läßt einen gewissen Unterschied erkennen, der durch Spuren von Halloysit neben Amesit und verschiedenen Eisenmineralen verstärkt wird (Bild 2). In diesem Zusammenhang soll vorerst hervorgehoben werden, daß die Tonfraktion der Probe 5 mehr Montmorillonit enthält als die Proben des Mittellahntales und des östlichen Schiefergebirgsrandes; darüber hinaus konnte Halloysit hier elektronenmikroskopisch zum ersten Male nachgewiesen werden.

\section{Das südliche Amöneburger Becken}

Das südliche Amöneburger Becken ist mit einem in der Regel mehrere Meter mächtigen Löß bedeckt. Nur die Talauen der Ohm, der Zwester Ohm und des 


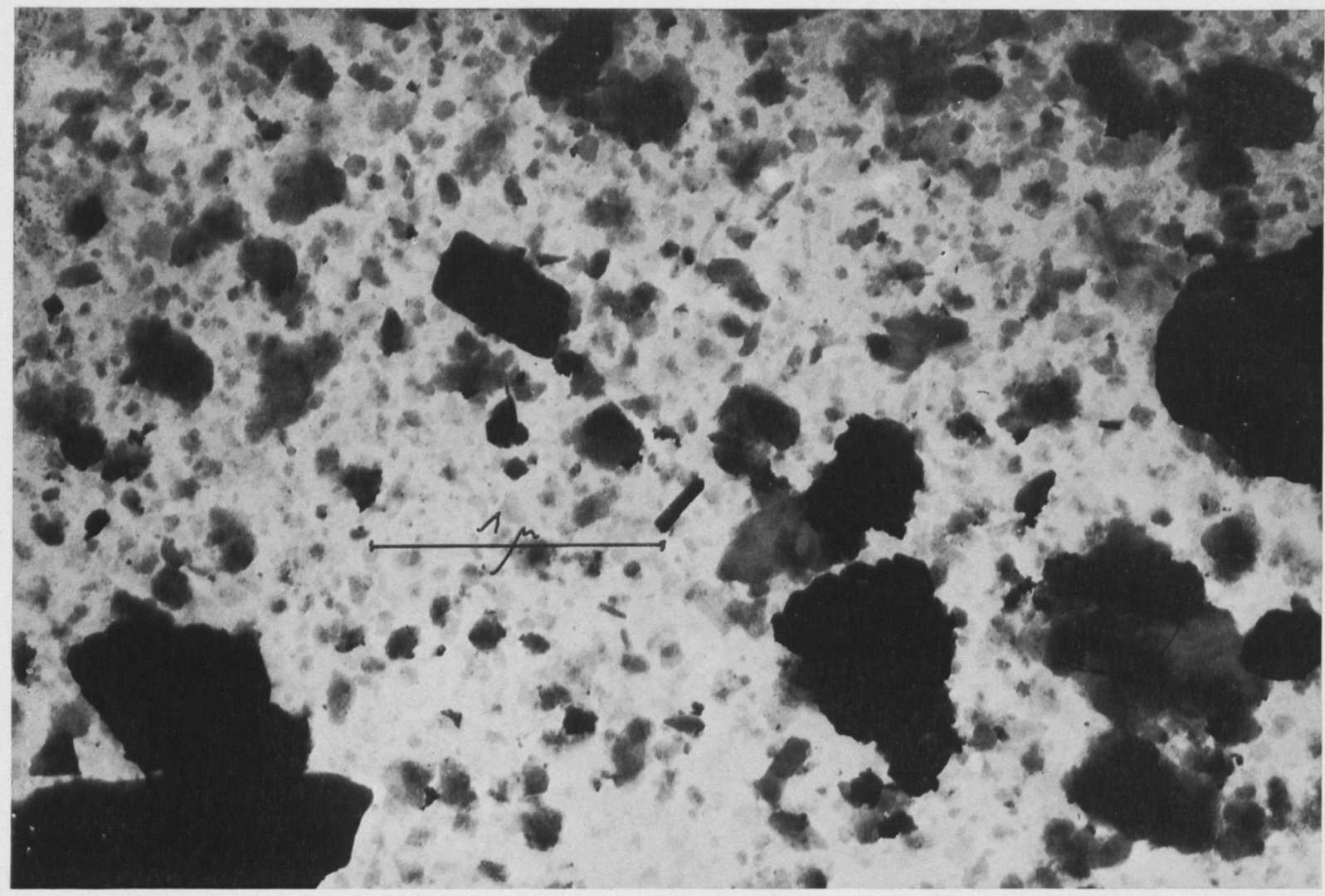

Bild 2: Elektronenmikroskopische Aufnahme der Tonfraktion $(<1 \mu)$ der Probe 5 (Kleinseelheim). Als Bestandteile sind zu erkennen, Illit, Hydroglimmer, Montmorillonit, Kaolinit, Halloysit, Quarz, Amesit und Eisenminerale.

Hunds-Baches sind lößfrei. Südlich der Linie Wittelsberg - Rauischholzhausen - Mardorf beginnt der Anstieg zu den miozänen Basaltdecken des nordwestlichen Vogelsberges, die hier von alttertiären Tonen und $\mathrm{z}$. T. quarzitisch verbackenen Sanden unterlagert werden.

Etwa $1 \mathrm{~km}$ nördlich Mardorf ist der Löß beiderseits der Straße nach Amöneburg aufgeschlossen. Die Probe 6 wurde hier dem rezenten Bv-Horizont entnommen. Probe 7 stammt aus der Peilschen Sandgrube östlich Wittelsberg; dort ist der Lößkomplex deutlich durch eine Steinsohle zweigeteilt. Die außerordentlich starke Verlehmung (ca. $35 \%$ Tongehalt), die Entwicklungstiefe, die intensive Färbung und der hohe Anteil an Kaolinit in der Tonfraktion weisen auf eine eemzeitliche Verwitterung des liegenden Lösses hin; der hangende Löß (oberhalb der Steinsohle) ist würmzeitlich und von einer holozänen Bodenbildung erfaßt worden; er lieferte das Material der Probe 7.

Die Laborbefunde dieser beiden Proben zeigen deutliche Abweichungen von den Analysendaten der zuvor beschriebenen Lösse. Innerhalb der Korngrößenverteilung sind die Maxima zwar auch in der für Lösse so typischen Fraktion $60-20 \mu$, doch sie sind weniger scharf ausgeprägt. Die feineren Fraktionen sind hier durchweg stärker vertreten. Es besteht kein
Zweifel, daß die Hauptmasse dieser Lösse auch aus Quarzstaub gebildet wird, aber ein Vergleich mit den Korngrößenverteilungen der anderen Lösse läßt vermuten, daß der Gehalt an Quarzkörnern gegenüber den Proben 1 bis 5 merklich abgenommen hat. Auch die mineralogische Zusammensetzung der Tonfraktion zeigt ein verändertes Bild. Neben Illit und Hydroglimmer ist - besonders in Probe 7 - Kaolinit in größeren Mengen anzutreffen. Ebenso tritt der Montmorillonit in den Röntgendiagrammen klar hervor, und die elektronenmikroskopischen Untersuchungen haben einen erhöhten Gehalt an Halloysit ergeben. Eisenminerale und Amesit scheinen als Spuren den Lössen des südlichen Amöneburger Beckens regelmäßig beigegeben zu sein.

\section{Rückschlüsse auf die Herkunftsgebiete der Würmlösse}

Die unterschiedliche Verteilung der verschiedenen Tonminerale in den Lössen des Marburger Raumes bekräftigt die Vermutung, daß das Lößmaterial nicht einem einheitlichen Ausblasungsgebiet zuzuschreiben ist; zumindest aber muß ein erheblicher Teil der Lösse aus petrographisch unterschiedlichen Gebieten herantransportiert worden sein, wobei die Möglichkeit nicht 
ausgeschlossen werden soll, daß darüber hinaus ein gewisser Anteil des Materials auch aus entfernteren Gegenden stammt; Hinweise dafür liefert ein Vergleich der tonmineralogischen Untersuchungen mit denen der Auelehme, worauf weiter unten einzugehen ist.

Bevor die einzelnen „Lößprovinzen“ und deren Liefergebiete betrachtet werden, sollen einige Bemerkungen zur Genese von Tonmineralen gemacht werden. Nach Degens $(1968,20-25)$ ist die Wirksamkeit der chemischen Reaktionen, die zur wäßrigen Dekomposition des Ausgangsmaterials und zur anschließenden Tonmineralneubildung führen, eine Funktion folgender Größen: 1. Ausgangsmaterial, 2. allgemeines Bildungsmilieu ausgedrückt in chemischer Zusammensetzung, $\mathrm{pH}$, Eh und Temperatur, und 3. Wasserkreislauf, d. h. Fortführung von gelöstem Material und Zufuhr von $\mathrm{CO}_{2}$-angereichertem Regenwasser. Einige Parameter unter 2. und 3. wie Temperatur, Niederschlag und Feuchtigkeit werden gewöhnlich als klimatische Einflüsse zusammengefaßt.

Das Material der o. a. Proben wurde jeweils den verwitterten B-Horizonten entnommen. Daher darf man annehmen, daß der überwiegende Teil der Tonminerale erst nach der Sedimentation gebildet worden ist. Da die Neubildung der Tonminerale unter gleichen - zumindest aber sehr ähnlichen - klimatischen Bedingungen stattfand, können die klimatischen Faktoren unberücksichtigt bleiben. Das Material war bei der Verwitterung Klimaschwankungen thermischer und/oder hygrischer Art in gleicher Weise ausgesetzt; darüber hinaus ist der Zeitraum, der für die Tonmineralneubildung zur Verfügung stand, für alle Proben gleich anzusetzen, da es sich um Würmlösse handelt, bei denen eine intensive Bodenbildung mit einer deutlich ausgeprägten Horizontierung erst nacheiszeitlich einsetzte. Auch die gleichen physikalischen Eigenschaften des unverwitterten Ausgangsmaterials (Löß) sollen in diesem Zusammenhang erwähnt werden. Aus all dem ergibt sich, daß die Unterschiede in der Tonmineralgarnitur der Lösse im Marburger Raum auf ein petrographisch unterschiedliches Ausgangsmaterial zurückzuführen sind.

Obgleich der heutige Zustand der Tonmineralzusammensetzung nicht ausschließlich von der Anwehung aus einem bestimmten Liefergebiet und von der Einwirkung des postsedimentären Klimas abhängt - es muß auch mit präsedimentären Verwitterungseinflüssen während der Loslösung der Komponenten aus dem primären Gesteinsverband und während aller Umlagerungsvorgänge vom Zeitpunkt der Abtragung an gerechnet werden (vgl. Kallenbach 1966, $600 \mathrm{f}$.) -, so zeigen doch die vorliegenden Ergebnisse der Analysen eine unmittelbare Abhängigkeit von ganz bestimmten Gesteinsprovinzen. Diese Abhängigkeit des Löß-Mineralbestandes von den Liefergebieten wurde - allerdings für wesentlich größere Räume und weniger differenziert - von verschiedenen Auto- ren beobachtet bzw. vermutet (so u. a. von E.-H. Müller 1959; BRUNNACKer 1954; DürR 1953; EdelMANN 1938; KALLENBACH 1966; SINDOWSKI 1938; WEIDENBACH 1952; ZöBELEIN 1940).

\section{Der Ostrand}

des Rheinischen Schiefergebirges

Für die Proben 1 und 2 (Elnhausen und Wehrshausen) aus dem petrographischen Bereich der paläozoischen Schiefergebirgsgesteine sind Hydroglimmer und Quarz typisch als Hauptbestandteile der Tonfraktion. Es fällt auf (Abb. 3), daß diese Proben einen wesentlich höheren Gehalt an Hydroglimmern aufweisen als das Lößmaterial aus dem Mittellahntal und dem Amöneburger Becken. Die Hydroglimmer treten als $Z$ wischenstufe beim Glimmerabbau auf, d. h. die Tonminerale liegen hier nicht als reine Endglieder vor, sondern als Übergangs- oder Wechsellagerungsminerale (Mixed-layers). Aus chemischen und strukturellen Gründen erscheinen Glimmer, besonders Biotit, als Ausgangsmaterial für diese Minerale besonders geeignet (SCHEFfer UND SCHAChtschabel 1966, 59 f.; Degens 1968,27$)$, d. h. aber, daß die Lösse von Elnhausen und Wehrshausen relativ reich an Glimmern gewesen sein müssen. Eine Untersuchung des nicht verwitterten Materials bestätigt diese Vermutung. Weiterhin fällt auf, daß in den Proben 1 und 2 der Illit, der in der Regel das vorherrschende Mineral der Tonfraktion mitteleuropäischer Lösse ist, nur eine untergeordnete Rolle spielt, während er in den Lössen des Mittellahntales und des Amöneburger Beckens deutlich den ersten Platz unter den Tonmineralen einnimmt.

Das reichliche Auftreten der Glimmer bzw. der Hydroglimmer als deren Verwitterungsprodukte spricht für das Rheinische Schiefergebirge als Liefergebiet der Lösse; natürlich muß angenommen werden, daß auch aus den Schottern der aus dem Schiefergebirge austretenden Bäche Material ausgeblasen wurde. Die Gesteine des östlichen Schiefergebirges verhielten sich bei einer periglazialen Verwitterung, wie sie zur Zeit der Lößbildung im Würm-Glazial herrschte, durchaus unterschiedlich. Vorwiegend die glimmerhaltigen Tonschiefer und paläozoischen Sandsteine lieferten das Ausgangsmaterial für den Löß in diesem Raum, denn gerade bei diesen Sedimentgesteinen und deren Abtragungsprodukten auf sekundären Lagerstätten (Flußschotter) bewirkte eine intensive Frosteinwirkung eine Zerkleinerung der Mineralkörner, insbesondere der Glimmer und Feldspäte (vgl. Scheffer, Meyer und Kalk 1958; auch: MartinI 1967). In diesem Zusammenhang soll nicht unerwähnt bleiben, daß die Annahme, Lößmaterial werde allein durch Frostsprengung bereitet, durchaus nicht bewiesen ist; Tricart (1956) stellte fest, daß trockener Frost praktisch wirkungslos ist und auf den Körnungsbereich des Lösses keinen Einfluß ausübt; vielmehr scheint eine markante Abhängigkeit der „Frostspren- 
gung" von dem Temperaturgang und der Durchfeuchtung und Wiedertrocknung zu bestehen; so finden sich auch zahlreiche Hinweise auf eine hochsommerliche Bodenaustrocknung in periglazialen Gebieten (vgl. u. a. Büdel 1959, 1960, 1962; POSER 1936; GRIPP 1929), und experimentelle Untersuchungen hinsichtlich der Frostverwitterung (MARTINI 1967) zeigen unterschiedliche Resultate in Abhängigkeit von den Feuchtigkeitsverhältnissen.

Kieselschiefer, quarzitische Sandsteine und Quarzite des Schiefergebirges kommen für die Bereitstellung des Lößmaterials nur bedingt in Betracht, wenn man die Bildung der Lößfraktion nicht allein auf die Frostsprengung zurückführen will. Diabase stehen in dem hier betrachteten Gebiet nicht in unmittelbarer Nähe an; sie sind oft recht mürbe, und die einzelnen Minerale sind teilweise von der Verwitterung stark angegriffen; da die Diabase am Ostrand des Schiefergebirges keine große Verbreitung zeigen, sollen sie hier nicht weiter berücksichtigt werden. Hinweise für eine Beteiligung von Diabasmaterial an den Lössen bei Elnhausen und Wehrshausen konnten nicht gefunden werden.

Zusammenfassend kann auf Grund der Ausführungen und Uberlegungen festgestellt werden, daß das Lößmaterial am Ostrand des Rheinischen Schiefergebirges vorwiegend von den Hängen des östlichen Gebirgsabfalles, aber auch aus den Schotterfluren der aus dem Gebirge austretenden Bäche ausgeweht worden ist.

\section{Das Mittellahntal}

Die Lösse des Mittellahntales, für die die Probe 4 (Gisselberg) charakteristisch ist, zeigen eine Verschiebung der Tonmineralzusammensetzung zugunsten des Illits (Abb. 3, Tab. 2 und Bild 1). Man darf annehmen, daß die Illite des Bv-Horizontes zum größten Teil aus Glimmern hervorgegangen sind, und zwar aus dem dioktraedrischen Muskovit und dem trioktraedrischen Biotit (vgl. Scheffer UND SCHACHTSCHABEL 1966, 52 und 62; Degens 1968, 26 f.; auch Rohdenburg und Meyer 1966, 73 ff.). Demnach muß das Lößmaterial, das bei Gisselberg sedimentiert wurde, glimmerhaltiger gewesen sein als die Lösse vom Ostrand des Schiefergebirges. Der relativ hohe Glimmeranteil läßt sich durch eine selektive Auswehung aus den Flußschottern der Mittellahn erklären, denn die Glimmerplättchen neigen eher $\mathrm{zu}$ einer äolischen Umlagerung als andere besser gerundete Komponenten. Außerdem werden sie bei der fluviatilen Sedimentation bevorzugt $\mathrm{n} \mathrm{a} \mathrm{ch}$ den ,runden“ Körnern abgelagert, so daß bei nachfolgender Ausblasung relativ viele Glimmer zur Verfügung stehen. Dieser Vorgang konnte sich während des Würm-Glazials in der Lahnschotteraue of wiederholen, nicht jedoch in einem Gebiet, in dem der Löß vorwiegend dem Verwitterungsmaterial der Hänge entstammt, wie z. B. am Ostrand des Rheinischen Schiefergebirges.
Interessant ist das Auftreten von Sarospatakit und Sepiolith im Löß von Gisselberg. Beide Minerale wurden weder in den Lössen am Ostrand des Schiefergebirges noch in den Lössen des Amöneburger Bekkens gefunden. Vergleichsuntersuchungen haben gezeigt, daß Sepiolith in Spuren in den Sedimentgesteinen des Buntsandsteins zu finden ist; in größeren Mengen tritt Sarospatakit in den intensiv gebleichten Zonen des Buntsandsteins auf, was für die Annahme einer hydrothermalen Bleichung des Sandsteins spricht (vgl. Heine 1970b; Hölting und Stengel-RutKowsKi 1964, 20 f.). Da Sarospatakit und Sepiolith, die nachweislich den Einfluß des Buntsandsteinmaterials am Aufbau der Lösse dokumentieren, nur im Löß des Mittellahntales angetroffen wurden, geht man bei der Interpretation der Ergebnisse sicherlich nicht $\mathrm{zu}$ weit, wenn man folgert, daß auch im Mittellahntal die Lößausblasung und -anwehung zum großen Teil auf engstem Raum stattfand. Gleichzeitig aber deutet dieser Befund darauf hin, daß das Lößmaterial in erster Linie aus den Lahnschottern ausgeblasen wurde, denn anderenfalls müßten die Lösse des Amöneburger Beckens, das im Westen, Norden und Osten von Buntsandsteingebieten umgeben ist, ebenfalls Sepiolith und/oder Sarospatakit enthalten. Auch die Lösse des Amöneburger Beckens stammen - wie weiter unten gezeigt wird - überwiegend aus Flußschottern.

Der Löß bei Goßfelden (Probe 4) gehört aufgrund der Laborergebnisse nicht mehr zum "Faziesbezirk“ des Mittellahntales. Röntgenanalysen und elektronenmikroskopische Aufnahmen veranschaulichen die enge Verwandtschaft dieses Lösses hinsichtlich der Tonmineralvergesellschaftung mit den Lössen am Ostrand des Schiefergebirges. Die Untersuchungen der Fraktionen $>120 \mu$ bestätigen diese Annahme. Während das gröbere Material des Gisselberger Lösses häufig rötlich gefärbte Quarzkörner des Buntsandsteins enthält, finden sich im Löß bei Goßfelden Quarzkörner in den gröbsten Fraktionen äußerst selten; sie sind schlechter zugerundet und tragen auch nicht die typischen rötlichen Eisenkrusten der Buntsandsteinquarzkörner. Daneben treten Gesteinsbruchstücke des Schiefergebirges deutlich hervor. Als Herkunftsgebiet des Lößmaterials kommt daher das östliche Rheinische Schiefergebirge und vor allem das Lahntal in diesem Bereich in Frage. Für eine Auswehung des Lößmaterials aus dem Lahntal sprechen auch die Röntgenanalysen. Zwischen 10 und $14 \AA$ sind die Reflexe sowohl bei Probe 4 (Goßfelden) als auch bei Probe 3 (Gisselberg) doppelt ausgebildet. Bei beiden Proben handelt es sich um Lösse am Rande des Lahntales. Hier scheint der Glimmerabbau stärker als bei den Proben 1 und 2 (Ostrand des Schiefergebirges) zu sein. Der Grund dafür liegt im unterschiedlichen Herkunftsgebiet. Das Material der Lösse von Goßfelden und Gisselberg stammt aus den Lahnschottern, Abtragungsprodukten somit, die bereits längere Zeit den verschiedensten Umlagerungs- und Verwitterungsprozessen ausgesetzt 
waren; präsedimentäre Verwitterungseinflüsse (einsetzender Glimmerabbau) haben dazu geführt, daß der Löß im Lahntal nach seiner Akkumulation einen weiter fortgeschrittenen Glimmerabbau zeigt als die Lösse des östlichen Rheinischen Schiefergebirges.

Der elektronenmikroskopisch ermittelte Unterschied der Löß-Proben aus dem Lahntal erklärt sich durch den unterschiedlichen petrographischen Aufbau der Lahnschotter (vgl. LANG 1955; Heine 1970a). Bei Goßfelden (Probe 4) sind einzig und allein Schiefergebirgskomponenten am Aufbau der Sedimente der Talfüllung beteiligt, während sich bei Gisselberg (Probe 3) Material aus dem Buntsandsteingebiet und - in unbedeutenden Mengen - aus dem Vogelsberg hinzugesellt.

\section{Das nördliche Amöneburger}

\section{B e cken}

Man sollte annehmen, daß sich die Verteilung der Tonminerale in den Lössen aus dem Amöneburger Becken nicht wesentlich unterscheidet. Hinsichtlich der qualitativen Tonmineralzusammensetzung ist das auch der Fall; Illit und Hydroglimmer sind vorherrschend; im südlichen Amöneburger Becken gesellt sich der Kaolinit noch zu ihnen. Montmorillonit und Quarz sind in allen Proben enthalten, doch zeigt das Material aus dem südlichen Gebiet einen bedeutend höheren Anteil an Montmorillonit (Abb. 3). In Spuren treten Halloysit, Amesit und Eisenminerale auf.

Die charakteristischen Tonminerale dieser Proben (5 bis 7) sind Montmorillonit und Halloysit. Der Montmorillonit bildet sich vorwiegend bei Anwesenheit von $\mathrm{Mg}$ - und $\mathrm{Ca}-$ Ionen, daher verwittern Plagioklase, Pyroxene und Amphibole häufig zu Montmorilloniten (vgl. auch ENGELHARDT 1961, 472; SCHEFFER UND SChachtschabel 1966, 55 f. und 63). Feldspäte, Augite und Hornblenden jedoch sind Hauptbestandteile der Vogelsbergbasalte. Auch der Halloysit ist ein typisches Tonmineral für Verwitterungsprodukte vulkanischer Gesteine und Lockermassen, doch scheint seine Bildung bevorzugt unter subtropischen und tropischen Klimabedingungen stattzufinden (MüCKENhausen 1964; HostermanN 1960, 287 ff.; Maignien 1965, 85; Millot 1964; Jasmund 1955; u. a.).

Probe 5 aus einem Lößprofil im Nordteil des Amöneburger Beckens bei Kleinseelheim enthält sowohl Montmorillonit als auch Halloysit und dokumentiert somit den Einfluß des basaltischen Vogelsberges im Herkunftsgebiet. Andererseits zeigt das Röntgendiagramm gewisse Ahnlichkeiten mit den Diagrammen der Proben, die zum größten Teil aus Schiefergebirgsmaterial aufgebaut wurden. Dennoch ist ein äolischer Transport des Materials vom Schiefergebirge in das Amöneburger Becken auszuschließen. Die sedimentpetrographischen Untersuchungen der würmzeitlichen Flußschotter (LANG 1955, 85; HeINe 1970a), die große Teile des nördlichen Amöneburger Beckens während der letzten Eiszeit bedeckten und heute von
Auelehmen überlagert werden, zeigen, daß am Aufbau dieser fluviatilen Sedimente kaum Basaltmaterial, jedoch um so mehr paläozoische Komponenten aus dem Kellerwald beteiligt sind. Dazu tritt Material des Buntsandsteingebietes. Es liegt nahe, diese relativ weiten würmzeitlichen Schotterflächen als Ausblasungsgebiete des Lösses im nördlichen Amöneburger Becken anzunehmen. Die Untersuchungsergebnisse stützen diese Annahme. Daß die für das Buntsandsteingebiet charakteristischen Minerale Sarospatakit und Sepiolith hier nicht auftreten, obgleich das Auswehungsgebiet auch Buntsandsteinmaterial enthält, macht um so deutlicher, daß Sarospatakit und Sepiolith überwiegend den intensiv gebleichten Gebieten entstammen; Bleichungszonen befinden sich aber nicht in nennenswertem Umfang im Einzugsbereich der Wohra, die den größten Teil der Sedimente des nördlichen Amöneburger Beckens herantransportierte.

4. Das s üdliche A möneburger Becken

In den Lössen des südlichen Amöneburger Beckens (Proben 6 und 7) ist der Einfluß des Vogelsbergbasaltes auf Grund der qualitativen Zusammensetzung der Tonminerale noch deutlicher ausgeprägt. Besonders der Montmorillonitanteil hat erheblich zugenommen. Daneben ist auch ein verstärktes Auftreten von Kaolinit festzustellen. Diese Beobachtungen können nicht allein auf postsedimentäre Veränderungen des Lößmaterials unter den örtlichen Verhältnissen i. e. S. zurückgeführt werden, vielmehr ergibt sich auch hier eine Differenzierung nach dem ursprünglichen Mineralbestand gegenüber den Lössen aus dem nördlichen Amöneburger Becken. Als Liefergebiete kommen wieder die würmzeitlichen Schotterauen der Flüsse in Frage, für Probe 6 das mit jungpleistozänen Sedimenten ausgefüllte Senkungsgebiet bei Schweinsberg und für Probe 7 die Schotterfluren verschiedener kleiner Bäche am Nordrand der Basaltdecken. Zumindest für die Sedimente der Schweinsberger Depression steht fest, daß sie reich an basaltischem Vogelsbergmaterial sind (HEINE 1970a).

Darüber hinaus scheint ein Teil der Lößsedimente weiteren Liefergebieten zu entstammen, nämlich dem angrenzenden Bereich des nordwestlichen Vogelsberges und dem Südteil des Amöneburger Beckens selbst. Diese Annahme beruht auf den Laborbefunden. Einmal hat sich gezeigt, daß bei der Korngrößenverteilung die feineren Fraktionen einen größeren Anteil an der Gesamtmenge haben, zum anderen ist der hohe Kaolinitgehalt bemerkenswert; beides schließt eine Beteiligung eines präsedimentär bereits intensiv verwitterten Materials am Aufbau der Lösse von Mardorf́ und Wittelsberg nicht aus. Vielmehr ist auf Grund der vorliegenden Ergebnisse sehr wahrscheinlich, daß die Lösse des südlichen Amöneburger Beckens z. T. aus den Verwitterungsdecken der im Süden angrenzenden Basaltlandschaft ausgeblasen wurden; auch ältere, interglazialzeitlich verwitterte Lösse im südlichen 
Amöneburger Becken kommen als Liefergebiete in Betracht. Der verhältnismäßig hohe Kaolinitgehalt in den Proben 6 und 7 scheint demnach präsedimentären Verwitterungseinflüssen zuzuschreiben zu sein. Kaolinitreiche Bodenbildungen, die von der nacheiszeitlichen Bodenentwicklung nur schwach überprägt wurden, sind im Vorderen Vogelsberg mancherorts anzutreffen (vgl. Weyl 1967, 46 ff.; Schönhals 1954, $150 \mathrm{ff}$.$) .$

\section{Die Ursachen der Lößverteilung im Marburger Raum}

Aus der Beziehung von Herkunfts- und Ablagerungsgebiet äolischer Sedimente wird oft auf die Windverhältnisse zur Zeit der Ablagerung geschlossen (Schönhals 1953, 1957; Poser 1948, 1950; WoldSTEDT 1961, $189 \mathrm{ff}$. u. a.). Es soll im folgenden untersucht werden, ob die Untersuchungsergebnisse der Lösse Schlüsse auf die Richtung des Windes zulassen. Anderungen in der Korngrößenverteilung, wie sie im Löß vom Auswehungsgebiet zum Ablagerungsraum von SchöNHALs (1955) nachgewiesen wurden, konnten im Marburger Raum nicht festgestellt werden. Doch scheinen die Lösse in der Umgebung Marburgs auf Grund ihrer tonmineralogischen Eigenschaften gewisse Hinweise auf die Windrichtungen zu geben.

Obgleich die Lösse überwiegend die nach Osten orientierten Hänge bedecken, ist die Annahme, daß die Lösse von westlichen Winden im Windschatten der Berge angeweht wurden (vgl. Blume 1949, 66), für den hier betrachteten Raum nicht befriedigend. Bereits einleitend wurde erwähnt, daß bei Westwinden und einem damit verbundenen Ferntransport des Lößmaterials über das Rheinische Schiefergebirge hinweg die Täler des Schiefergebirges sowie die Ostabdachung desselben (Michelbach-Elnhäuser Tiefenzone im Westen Marburgs) und das Mittellahntal im Bereich des Buntsandsteins wesentlich mehr Lößsedimente enthalten müßten, als es in Wirklichkeit der Fall ist. Da sich aber während der Würmeiszeit die Windverhältnisse nicht wesentlich von den rezenten unterschieden, $d . h$. es wehten vorherrschend Winde aus westlicher Richtung, müssen andere Ursachen für die Lößverteilung im Marburger Raum verantwortlich sein. Auch die Einwirkung des Geländes in diesem stark reliefierten Gebiet vermag die Winde nur an wenigen Stellen stärker abzulenken (Klima-Atlas Hessen 1950; ERIKSEN 1966, 86). Wollte man die Verbreitung des Lösses allein von den Windverhältnissen abhängig machen, so müßten beispielsweise die Lösse des Amöneburger Beckens, deren Herkunftsgebiete durch die mineralogischen Untersuchungen bekannt sind, von wechselnden Winden aus nördlichen, östlichen und südlichen Richtungen ausgeweht worden sein. Die Windverteilung zeigt aber, daß Winde aus diesen Richtungen im Marburger Raum nur mit geringer Häufigkeit vertreten sind. Es stellt sich daher die Frage. warum die vor- herrschenden westlichen Winde einen so geringen Einfluß auf den äolischen Transport des Lößmaterials vom Ausblasungsgebiet zum Ablagerungsraum haben.

Bei der Betrachtung der geologischen Übersichtskarte von Hessen 1:300 000 (bearbeitet von F. RöSING) fällt auf, daß die Verbreitung und Mächtigkeit des Lösses von verschiedenen Faktoren abhängt. Zuerst einmal wird die Ablagerung und spätere Erhaltung des Lösses in den tiefer gelegenen Landschaften begünstigt, so in den Becken und Senken, vor allem in der Wetterau, im Taunus-Vorland, im Rheingau, im Limburger Becken, im Amöneburger Becken, in der Schwalm, in der Hessischen Senke zwischen Efze und Kassel etc. (vgl. Schönhals 1954, 54 ff.). Aber auch im Westerwald und im Vogelsberg kommen Lößdekken von beachtlicher Ausdehnung vor. Mehr oder weniger lößfrei sind die ausgedehnten Gebiete des mittleren und unteren Buntsandsteins. Auch im Rheinischen Schiefergebirge und im Kellerwald sind Lößablagerungen von nennenswertem Umfang selten oder sie fehlen ganz. Es soll in diesem Zusammenhang nicht auf weitere Einzelheiten eingegangen werden; wichtig ist lediglich folgende Feststellung: Größere Lößablagerungen befinden sich stets (1) in der Nähe von Flußläufen, die im Pleistozän weite Schotterfluren besaßen und deren Einzugsgebiete von bestimmter petrographischer Beschaffenheit sind, und (2) im unmittelbaren Einzugsbereich von Gesteinen, die bei einer periglazialen Verwitterung relativ viel Material der SchluffFraktionen bereitstellen können.

$\mathrm{Zu} \mathrm{(1):} \mathrm{Die} \mathrm{Schotterfluren} \mathrm{größerer} \mathrm{Flußläufe} \mathrm{kön-}$ nen als Ausblasungsgebiete der Lösse angesehen werden, sofern die pleistozänen fluviatilen Sedimente aus einem Material aufgebaut werden, das unter kaltzeitlichen Verwitterungseinflüssen dazu neigt, einen gewissen Anteil an Teilchen in der Schluff-Fraktion zu bilden. Ist das nicht oder im abgeschwächten Maße der Fall, so kann auch keine Auswehung eines schluffigen Materials erfolgen. Beispiele dafür liefern alle Flußläufe, die ihr Einzugsgebiet im Unteren und Mittleren Buntsandstein haben, bzw. deren Lauf sich über längere Strecken im $s u$ und/oder $s m$ befindet. Dann nämlich bestehen die Flußschotter überwiegend aus den Abtragungsprodukten des Buntsandsteins; diese aber werden in der Regel aus feinkörnigen Sandsteinen und Quarzsanden gebildet; die pleistozäne Verwitterung vermochte nicht, die Quarzkörner, deren Durchmesser oft im Sandbereich und fast nie im Schluffbereich liegen, weiter zu zerkleinern, so daß Material für eine äolische Auswehung bereitstünde. Die geologische Karte zeigt viele Beispiele.

$\mathrm{Zu}$ (2): Auffällig ist, daß in der Nähe größerer Basaltgebiete Lößablagerungen häufig $z u$ finden sind (Westerwald, Vogelsberg, Hessische Senke). Der Grund liegt in der Eigenschaft der Basalte, bei der Verwitterung relativ viel Material im Korngrößenbereich $60-20 \mu \mathrm{zu}$ liefern (vgl. Kvitkovič 1968, 11 ff.; Sukhodrovski 1962, zit. nach Martini 1967, 
158 f.) ${ }^{2}$ ). Das gleiche gilt für den Oberen Buntsandstein und den Keuper (vgl. die geologische Ubersichtskarte!). Es soll hier nicht entschieden werden, ob das Lößmaterial den Verwitterungsdecken der Basalte, des Oberen Buntsandstein und des Keuper direkt vom Wind entnommen wurde, um an anderer - meist nicht weit entfernter - Stelle wieder abgelagert zu werden, oder ob das Verwitterungsmaterial vor der Lößausblasung eine Umlagerung erfahren hat, sei es in Form solifluidaler oder/und fluviatiler Bewegungen.

$\mathrm{Da}$ im östlichen Rheinischen Schiefergebirge der Löß keine große Verbreitung hat, obgleich die mineralogisch-petrographischen Voraussetzungen nicht ungünstig sind, mag daran liegen, daß in der Würmeiszeit die Gebirge bereits ihre heutige Oberflächengestalt aufwiesen, wodurch wegen der stärkeren Zertalung und der höheren Niederschläge die Abtragung der ohnehin nicht so mächtigen Lösse beschleunigt wurde. Die winterliche Schneedecke verhinderte dazu in den Gebirgen für eine wesentlich längere Zeit als in den tiefer gelegenen Regionen eine Lößbildung. Dennoch zeigen die Schuttdecken der hessischen Mittelgebirge eine deutliche äolische Komponente, wie Semmel $(1964,1966)$ feststellen konnte.

Bevor die Frage nach den Ursachen der Lößverbreitung im Marburger Raum wieder aufgegriffen wird, sind noch einige Bemerkungen zum äolischen Transport des Materials nötig.

Körner der Fraktion $<0,06 \mathrm{~mm}$ können schon bei mittleren Windgeschwindigkeiten schwebend transportiert werden. Sindowski (1956) führt ein Beispiel an, wo bei einer Windgeschwindigkeit von $6-12 \mathrm{~m} / \mathrm{sec}$ in $10 \mathrm{~cm}$ Höhe Quarzkörner über $0,5 \mathrm{~mm} \phi$ rollend, von $0,5-0,1 \mathrm{~mm} \phi$ springend und $<0,06 \mathrm{~mm} \phi$ schwebend transportiert wurden. Voraussetzung dafür ist aber, daß das Material in trockenem Zustand vorliegt. Eine Materialauswehung kann demnach nur stattfinden, wenn eine gewisse Windgeschwindigkeit herrscht und wenn die Schotter bzw. die Verwitterungsdecken, aus denen die Teilchen vom Wind aufgenommen werden, zuvor oberflächlich völlig ausge-

2) MARTINI (1967) konnte experimentell nachweisen, daß verschiedene Gesteine unterschiedlich auf die Frostverwitterung reagieren. Vulkanite, insbesondere Tertiär-Basalte, sind gegenüber der Frostverwitterung sehr resistent. Dahor liegt die Vermutung nahe, daß in den Basaltgebieten (Vogelsberg, Westerwald) nicht kaltzeitliche Verwitterungsprozesse den mengenmäßig größten Anteil des - später äolisch umgelagerten - Materials lieferten, sondern daß in den Basaltgebieten vorwiegend ältere Verwitterungsdecken das Feinmaterial bereitstellten; fossile, präpleistozäne Böden (z. T. sind sie umgelagert) sind auch heute noch im Vogelsberg und Westerwald oft anzutreffen; sie konnten von den eiszeitlichen Abtragungsvorgängen nicht restlos entfernt werden. Die Tonmineraluntersuchungen der Lösse des südlichen Amöneburger Beckens, deren Hauptliefergebiet der Vordere Vogelsberg ist, deuten ebenfalls auf Grund ihres erhöhten Kaolinitgehaltes darauf hin, daß ein Teil des Lößmaterials älteren (z. T. präpleistozänen) Böden entstammt. trocknet sind (vgl. Woldstedt 1961, 178). Die Voraussetzungen der Auswehung von Lößstaub waren während der Glazialzeiten nur bei besonderen Wetterlagen gegeben. $\mathrm{Ob}$ die vorherrschenden Westwinde, die meist feuchte Luftmassen heranführen, die Ausblasung und den Transport des Lößmaterials besorgten, ist daher zumindest zweifelhaft. Schönhals (1953) konnte z. B. zeigen, daß bei Hradec Králové (Königgrätz) die Lösse von Winden aus östlicher Richtung herantransportiert wurden, obgleich in Nordböhmen Winde aus westlichen Richtungen vorherrschen und wohl auch auf Grund der Untersuchungen von Poser (1948, 1950; vgl. auch Woldstedt 1961, 190 ff.) für das Würm-Glazial anzunehmen sind. Die Vermutung liegt nahe, daß die nordböhmischen Lösse deshalb von östlichen Winden ausgeweht wurden, weil mit ihnen eine Austrocknung der Hochflutbetten der Flüsse einherging. Demnach scheinen hier nicht die allgemein vorherrschenden Winde für den Lößtransport verantwortlich zu sein.

Vermutlich wurde auch im Marburger Raum der Transport des Lößstaubes nicht in erster Linie von den vorherrschenden Westwinden vorgenommen, sondern von Winden aus unterschiedlichen Richtungen. Entscheidend für die Ausblasung des Materials war in der Marburger Landschaft nicht die Windrichtung, sondern vielmehr eine (sommerliche) Austrocknung der Liefergebiete. Daß dennoch fast ausschließlich Material der Fraktionen 0,06-0,01 mm äolisch verfrachtet worden ist - trotz der oft geringen Entfernungen zwischen Herkunfts- und Sedimentationsgebiet -, scheint folgende Gründe zu haben: (1) Die Winde waren nicht stark genug, um auch gröberes Material (Sand) zu transportieren, wie das in Gebieten mit Flugsandfeldern und Binnendünen der Fall war. Die Reliefverhältnisse der Marburger Landschaft haben sicherlich mit dazu beigetragen, daß länger anhaltende starke Winde bei gleichzeitiger Austrocknung der Ausblasungsgebiete nicht morphologisch wirksam werden konnten. (2) Feineres Material $(<0,01 \mathrm{~mm} \phi)$ liegt im ausgetrockneten Zustand nicht mehr in Form von Primärteilchen vor, sondern die Einzelkörner sind miteinander verklebt. Durch Frosteinwirkung und den Wechsel der Durchfeuchtung kann eine Zerkleinerung und damit die Ausbildung kleiner Aggregate begünstigt werden. Die Untersuchung der pleistozänen fluviatilen Sedimente im Marburger Raum (Heine 1970a) hat jedoch gezeigt, daß feinschluffige und tonige Ablagerungen äußerst selten sind und daher als Auswehungsmaterial kaum in Frage kommen. Lediglich die Lösse im südlichen Amöneburger Becken (Proben 6 und 7) lassen auf Grund der Korngrößen- und tonmineralogischen Zusammensetzung erkennen, daß hier feinschluffiges und toniges Material sedimentiert wurde, das den basaltischen Verwitterungsdecken des Vorderen Vogelsberges entstammt (vgl. Anm. 2).

Sicherlich wechselten die Ausblasungsräume der Würmlösse bei Marburg nicht nur räumlich, sondern 
auch zeitlich. Dennoch lassen sich - wie die Untersuchungen und Úberlegungen gezeigt haben - zumindest für den größten Anteil des Lößmaterials Auswehungs- und Akkumulationsgebiete zueinander in Beziehung setzen. Die Abtragungs-, Transport- und Sedimentationsvorgänge haben sich auf engstem Raum abgespielt. Für die Lösse am Ostrand des Rheinischen Schiefergebirges ergeben sich als Liefergebiete die Schotterfluren der aus dem Gebirge austretenden Bachläufe, dazu die Hänge des Gebirgsabfalles; die Lößvorkommen an den Hängen des Mittellahntales entstammen zu einem erheblichen Teil den würmzeitlichen Lahnschottern; die Lösse im nördlichen Amöneburger Becken wurden aus der Schotteraue der Ohmtaldepression ausgeblasen, dazu mag auch aus südlicher Richtung vom Vorderen Vogelsberg Material herantransportiert worden sein; als Liefergebiete der Lösse im südlichen Amöneburger Becken werden die Hochflutbetten der aus dem Vogelsberg kommenden Flüsse und die Verwitterungsdecken des Vorderen Vogelsberges angesehen.

Die auffällige Verbreitung der Lösse an den nach Osten gerichteten Hängen hat nicht seine Ursache in den vorherrschenden Westwinden und einer damit verbundenen Ablagerung im Windschatten topographischer Hindernisse, sondern ist vielmehr auf Winde aus östlichen und südlichen Richtungen zurückzuführen. Natürlich ist nicht ausgeschlossen, daß eine ursprünglich geringmächtige Lößdecke auch die Westhänge bedeckte, daß jedoch auf den östlichen Talseiten infolge regenbringender Westwinde die Lößschleier wieder entfernt wurden (vgl. Blume 1949, 66). Vermutlich fand schon während der Lößanwehung oder später auf den nach Westen exponierten Hängen eine Verlagerung und Abtragung des lockeren und dünnen Lößschleiers statt (vgl. SchöNHALs 1954, 54).

\section{Abschließender Vergleich der Ergebiiisse mit Untersuchungen an Auelebmen}

Die Auelehme erfüllen die Täler der Lahn und ihrer Nebenflüsse. Im allgemeinen ist - wie im Lahntal eine deutliche Abnahme der Auelehmmächtigkeit flußaufwärts festzustellen. Junge tektonische Bewegungen können jedoch die Auelehmsedimentation stark beein-

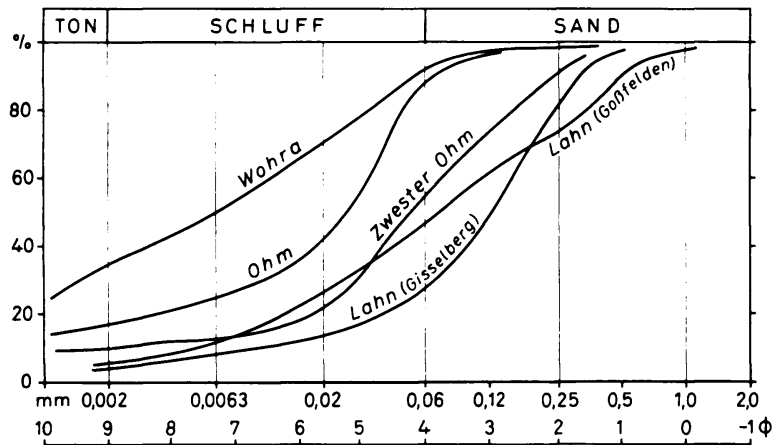

$A b b .4$ : Kornsummenkurven verschiedener Auclehme flussen. Die größten Mächtigkeiten weist das Amöneburger Becken im Bereich der Ohmtaldepression auf (bis $5,2 \mathrm{~m}$ ). Der Beginn der Auelehmsedimentation fällt im Lahntal südlich Marburgs und im nördlichen Amöneburger Becken in die Allerödzeit (LANG 1954, 1955; KOCKel 1958).

Neben der granulometrischen Analyse (Abb. 4) zeigt die mineralogische Zusammensetzung der Tonfraktion der Auelehme eine Abhängigkeit von den jeweiligen Einzugsgebieten. Im Lahntal sind - besonders im Bereich des Schiefergebirges - die 14 Å-Minerale stark vertreten (Abb. 5), daneben sind Quarz, Illit und

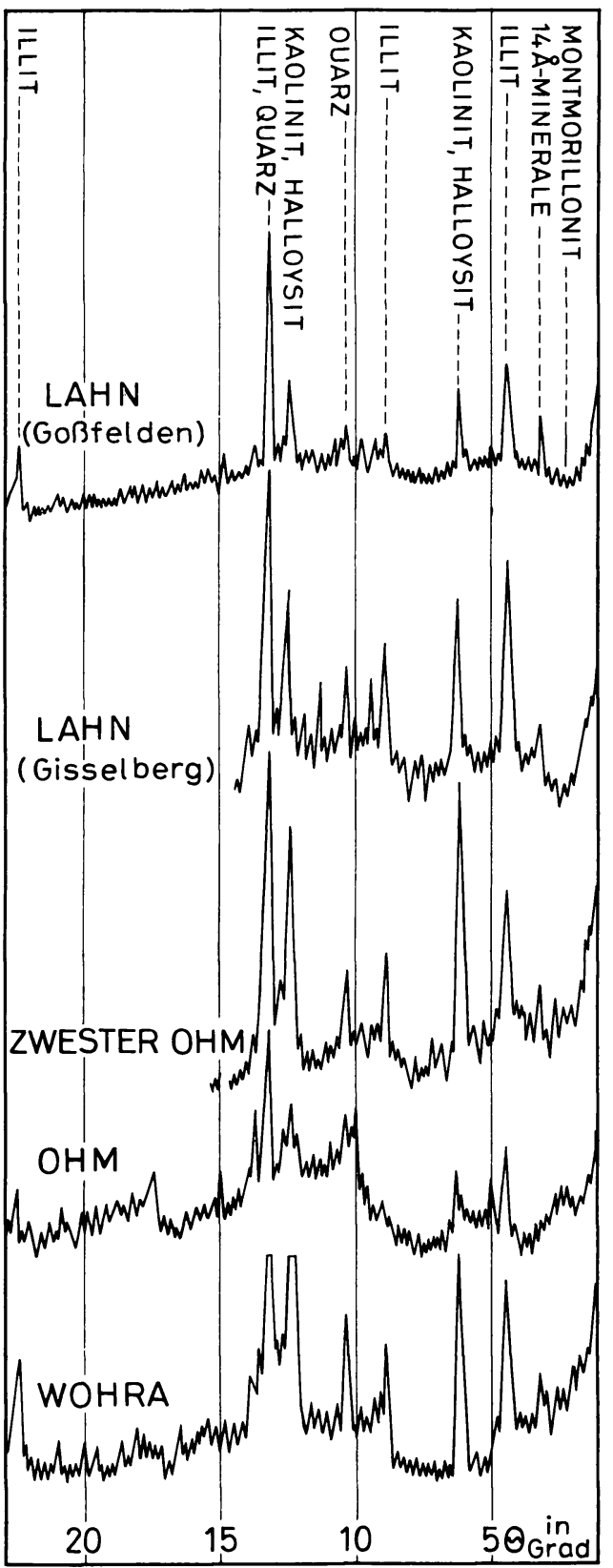

Abb. 5: Röntgendiagramme verschiedener Auelehme 
Kaolinit zu erkennen. Das Röntgendiagramm der Wohra-Auelehme zeigt analoge Verhältnisse; daraus ist zu schließen, daß der sehr feinkörnige Auelehm der Wohra zu einem gewissen Teil aus Verwitterungsund Abtragungsmaterial des Kellerwaldes hervorgegangen ist. Bei der $\mathrm{Zwester} \mathrm{Ohm}$ sind es die mesozoischen und tertiären nichtvulkanischen Sedimente, die das Bild bestimmen, doch macht sich der nahe Vogelsberg (Basalt) durch ein verstärktes Auftreten von Montmorillonit und Halloysit bereits bemerkbar. Dieser basaltische Einfluß (Montmorillonit, Halloysit) bestimmt das Bild des Röntgendiagramms und der elektronenmikroskopischen Aufnahme eines Auelehms aus dem Amöneburger Becken. Elektronenmikroskopische Untersuchungen ergaben, daß der Holloysit nur in den Auelehmen zu finden ist, die Vogelsbergmaterial enthalten (HeINe 1970a).

Diese enge Beziehung der Auelehme zu dem petrographischen Aufbau der jeweiligen Einzugsgebiete der Flüsse macht deutlich, daß das Auelehmmaterial zum größten Teil den Verwitterungsdecken der höher gelegenen Gebiete entstammt; gleichzeitig aber auch unterstreichen die Ergebnisse der Auelehmuntersuchungen wegen ihrer Ubereinstimmung mit den Analysendaten der Lößproben die Feststellung, daß in der Marburger Landschaft Lößauswehung und Lößablagerung auf engstem Raum stattfand und daß auf Grund der Tonmineralgarnituren verschiedenen Lössen verschiedene Herkunftsgebiete zugeordnet werden können. Allerdings muß einschränkend hinzugefügt werden, daß sich zur Zeit keine quantitativen Angaben machen lassen; daher kann nicht bestimmt werden, welche Materialmengen die einzelnen Lösse aus den verschiedenen Lieferräumen erhielten. Wäre jedoch beim Transport eine wesentliche Vermischung des Lößstaubes verschiedener Auswehungsgebiete erfolgt, dann hätte sich das auf die qualitative Zusammensetzung der Tonminerale auswirken müssen.

\section{Literatur}

Beutelspacher, H., und van der Marel, H. W. (1968): Atlas of Electron Microscopy of Clay Minerals and their Admixtures. Amsterdam.

BlanCKENHORN, M. (1930): Erläuterungen zur Geol. Karte von Preußen etc. Lieferung 299, Blatt Amöneburg - Homberg a. d. Ohm. Berlin.

Blume, H. (1949): Die Marburger Landschaft. Marburger Geogr. Schr., Bd. 1, Marburg.

Brown, G. (Editor, 1961): The X-ray identification and crystal structures of clay minerals. Mineralogical Society, London.

BrunNACKer, K. (1954): Löß und diluviale Bodenbildungen in Südbayern. Eisz. u. Gegenw. 4/5, 83-86.

Büdel, J. (1959): Periodische und episodische Solifluktion im Rahmen der klimatischen Solifluktionstypen. Erdkde., XIII, 297-314.

- (1960): Die Frostschuttzone Südost-Spitzbergens. Colloquium Geographicum 6.
- (1962): Die Abtragungsvorgänge auf Spitzbergen im Umkreis der Barentsinsel. Verh. d. 33. Dt. Geogr.-Tag Köln, 337-375, Wiesbaden.

Degens, E. T. (1968): Geochemie der Sedimente. Stuttgart.

DürR, F. (1953a): Zusammenhang zwischen Bodengenetik und Schwermineralführung. Diplomarbeit Kiel.

- (1953b): Leitmineraluntersuchung. In: GüEnther: Feinstratigraphische Studien III. N. Jb. Geol. Pal. Mh., 97-111.

EdelmanN, C. H. (1938): Ergebnisse der sedimentpetrographischen Forschung in den Niederlanden und den angrenzenden Gebieten. 1932 und 1937. Geol. Rdsch., 29, 223-273.

EngelhardT, W. von (1961): Neuere Ergebnisse der Tonmineralforschung. Geol. Rdsch., 51, 457-477.

ERIKSEN, W. (1966): Klima und Witterung im Raume Marburg. In: Marburger Geogr. Schr. 30, 79-90, Marburg.

GRIM, R. E. (1954): Clay mineralogy. New York.

GrIPP, K. (1929): Glaciologische und geologische Ergebnisse der Hamburgischen Spitzbergen-Expedition 1927. In: Abh. auf d. Gebiete d. Naturw. Hrsg. vom Naturw. Ver. in Hamburg, Bd. XXII, H. 3-4.

Guenther, E. W. (1961): Sedimentpetrographische Untersuchungen an Lössen. Fundamenta (Monographien zur Urgeschichte), Reihe B, Bd. 1, Köln.

Heine, K. (1970a): Fluß- und Talgeschichte im Raum Marburg. (Eine geomorphologische Studie.) Bonner Geogr. Abh. 42.

- (1970b): Die Bleichung der Sandsteine bei Marburg/ Lahn - eine hydrothermale Bildung. Notizbl. hess. L.-Amt Bodenforsch., 98, 1970 (im Druck).

Hölting, B., und Stengel-Rutkowski, W. (1964): Beiträge zur Tektonik des nordwestlichen Vorlandes des basaltischen Vogelsberges, insbesondere des Amöneburger Beckens. Abh. hess. L.-Amt Bodenforsch., 47, Wiesbaden.

Hostermann, J. W. (1960): Geology of the clay deposits in parts of Washington and Idaho. In: Clay and Clay Minerals. Proc. 7th Nat. Conf. on Clays and Clay Minerals, 285-292, Washington.

Jacobshagen, E. (1933): Fossile Reste der Mammut-Fauna aus Marburg und aus anderen Orten der Provinz Hessen-Nassau. Sitz.-Ber. Ges. z. Beförderung d. gesamten Naturw. zu Marburg, 68, H. 1, 1-32, Berlin.

Jasmund, K. (1955): Die silikatischen Tonminerale. Weinheim.

Kallenbach, H. (1966): Mineralbestand und Genese südbayerischer Lösse. Geol. Rdsch., 56, 726-748.

Klima-Atlas von Hessen, Bad Kissingen 1950.

Kockel, C. W. (1958): Schiefergebirge und Hessische Senke um Marburg/Lahn. Sammlung Geol. Führer, Bd. 37, Berlin.

Kvitkovič, J. (1968): Die geomorphologischen Verhältnisse im NO-Teil des ostslowakischen Tieflandes. Würzburger Geogr. Arb., H. 22/III.

LANG, H. D. (1954): Ein Alleröd-Profil mit eingelagertem Laacher-See-Tuff bei Marburg/Lahn. N. Jb. Geol. Paläont., Mh. 8, 362-372.

- (1955): Zur Flußgeschichte der Lahn. Ergebnis geröllanalytischer Untersuchungen in der Umgebung von Marburg/Lahn. Diss. Marburg, unveröff.

LožEK, V. (1965): Das Problem der Lößbildung und die Lößmollusken. Eisz. u. Gegew. 16, 61-75.

Maignien, R. (1966): Review of research on laterites. Natural resources research IV, UNESCO, Paris. 
Martini, A. (1967): Preliminary experimental studies on frost weathering of certain rock types from the West Sudetes. Biuletyn Peryglacjalny Nr. 16, 147-194.

Millot, G. (1964): Géologie des argiles. Paris.

Mückenhausen, E. (1964): Das elektronenmikroskopische Bild verschiedener Bodentypen. 8th Intern. Congress of Soil Science, Bucharest, Romania, 1964, Vol. III, Commission VII (soil mineralogy), Paper 6, 1125-1133.

Müller, E.-H. (1959): Art und Herkunft des Lösses und Bodenbildungen in den äolischen Ablagerungen Nordrhein-Westfalens unter Berücksichtigung der Nachbargebiete. Fortschr. Geol. Rheinl. u. Westf., 4, 255-265, Krefeld.

Rohdenburg, H., und Meyer, B. (1966): Zur Feinstratigraphie und Paläopedologie des Jungpleistozäns nach Untersuchungen an südniedersächsischen und nordhessischen Lößprofilen. Sonderh. z. Tag. d. DEUQUA. Aus: Mitt. dt. Bodenkdl. Ges., Bd. 5, 1-135, Göttingen.

Poser, H. (1936): Talstudien in Westspitzbergen und Ostgrönland. Z. Gletscherkunde 24, 43-98.

- (1948a): Boden und Klimaverhältnisse in Mitteleuropa während der Würmeiszeit. Erdkde., II, 53-68.

- (1948b): Æolische Ablagerungen und Klima des Spätglazials in Mittel- und Westeuropa. Naturwiss. 35, 269-276 und 307-312, Berlin.

- (1950): Zur Rekonstruktion der spätglazialen Luftdruckverhältnisse in Mittel- und Westeuropa auf Grund der vorzeitlichen Binnendünen. Erdkde. IV, 81-88.

Scheffer, F., und Schachtschabel, P. (1966): Lehrbuch der Bodenkunde. Stuttgart.

Scheffer, F., Meyer, B., und Kalk, E. (1958): Mineraluntersuchungen am Würmlöß südniedersächsischer Lößfluren als Voraussetzung für die Mineralanalyse verschiedener Lößbodentypen. Chemie der Erde 19, 338-360.
Schönhals, E. (1953): Gesetzmäßigkeiten im Feinaufbau von Talrandlössen mit Bemerkungen über die Entstehung des Lösses. Eisz. u. Gegenw. 3, 19-36.

- (1954): Die Böden Hessens und ihre Nutzung. Abh. hess. L.-Amt Bodenforsch., 2, Wiesbaden.

- (1955): Kennzahlen für den Feinheitsgrad des Lösses. Eisz. u. Gegenw. 6, 133-147.

- (1957): Spätglaziale äolische Ablagerungen in einigen Mittelgebirgen Hessens. Eisz. u. Gegenw. 8, 5-17.

Semmel, A. (1964): Junge Schuttdecken in hessischen Mittelgebirgen. Notizbl. hess. L.-Amt Bodenforsch., 92, 275-285, Wiesbaden.

- (1966): UUber die Gliederung pleistozäner Schuttdecken in Hessen. Vortrag auf der 13. Tag. d. DEUQUA am 9. 8. 1966 in Göttingen.

SinDowski, K. H. (1938): Korngrößen- und Schwermineralverteilung in den Tuffen und Lössen des Laacher-SeeGebietes. Nr. 1. Decheniana 98. A. 55-71.

- (1956): Korngrößen und Kornformauslese beim Sandtransport durch Wind. (Nach Messungen auf Norderney.) Geol. Jb., 71, 517-526.

Tricart, J. (1956): Étude expérimentale du problème de la gélivation. Biuletyn Peryglacjalny Nr. 4, 285-318.

Weidenbach, F. (1952): Gedanken zur Lößfrage. Eisz. u. Gegenw. 2, 25-36.

WEYL, R. (1967): Geologischer Führer durch die Umgebung von Gießen. Gießen.

Woldstedt, P. (1961): Das Eiszeitalter. Grundlinien einer Geologie des Quartärs. Bd. 1: Die allgemeinen Erscheinungen des Eiszeitalters. Stuttgart.

ZöbelEIN, H. K. (1940): Geologische und sedimentpetrographische Untersuchungen im niederbayrischen Tertiär (Blatt Pfarrkirchen). N. Jb. Min. etc. Beil. Bd. 84 B, 233-302. 